\title{
Approximate Clustering of Noisy Biomedical Data
}

\author{
Krzysztof Boryczko and Marcin Kurdziel \\ Institute of Computer Science, AGH University of Science and Technology, \\ al. Mickiewicza 30, 30-059 Kraków, Poland \\ \{boryczko, kurdziel\}@agh.edu.pl
}

\begin{abstract}
Classical clustering algorithms often perform poorly on data harboring background noise, i.e. large number of observations distributed uniformly in the feature space. Here, we present a new density-based algorithm for approximate clustering of such noisy data. The algorithm employs Shared Nearest Neighbor Graphs for estimating local data density and identification of core points, which are assumed to indicate locations of clusters. Partitioning of core points into clusters is performed by means of Mutual Nearest Neighbor distance measure. This similarity measure is sensitive to changes in local data density, and is thus useful for discovering clusters that differ in this respect. Performance of the presented algorithm was demonstrated on three data sets, two synthetic and one real world. In all cases, meaningful clustering structures were discovered.
\end{abstract}

Keywords: Cluster analysis, Noisy data, Multidimensional data, Shared Nearest Neighbor Graph, Mutual Nearest Neighborhood.

\section{Introduction}

Formerly, research in cluster analysis focused on data sets were almost all observations are believed to be members of some clusters. Even if outlier observations were accounted for, they were thought as exceptions rather than significant fraction of the data set. In recent years however, efforts were made to develop clustering techniques suitable for data sets were outlier observations are so frequent that they in fact become a noisy background in which clusters are submerged. A classical example is the DBSCAN algorithm [1, which employs a density-based definition of clusters. Density-based notion of clusters was also adopted in [2]. Unlike DBSCAN, which relay on simple counting of points within spheres of some given radius, this method employ Shared Nearest Neighbor (SNN) graphs for density estimation. Some approaches to noisy data clustering employ data sampling instead of explicit density estimation. This is the case in CURE 3 algorithm for example. Yet another approach to this task focus on graph-based cluster connectivity measures. Typical representatives of this approach are Chameleon [4] and ROCK [5] algorithms.

M. Bubak et al. (Eds.): ICCS 2008, Part I, LNCS 5101, pp. 630640, 2008.

(C) Springer-Verlag Berlin Heidelberg 2008 
We present a new algorithm for clustering of high-dimensional, noisy data. The algorithm, named Clustering With Nearest Neighborhood (CWNN), is inspired by ideas presented in [2, 6] and [7. CWNN employs the SNN graph to detect the so-called core data points. This allows for explicit handling of background noise as well as automatic assessment of the number and shapes of clusters. The strength of the our approach lies in a method for partitioning core points into origins of clusters. We propose to partition the set of core points by employing the Mutual Nearest Neighbor (MNN) distance measure computed over the proximity measure derived from the SNN graph. Experimental results illustrating performance of this method for data harboring background noise, including multidimensional cases, are demonstrated. It is important to note here, that perfect discrimination between background noise and data clusters is often unattainable, especially if they are of comparable densities. Therefore, CWNN should be seen as an approximate clustering method.

\subsection{Mutual Nearest Neighbor Distance Measure}

Consider a set of points $X=\left\{\mathbf{x}_{1}, \mathbf{x}_{2}, \ldots, \mathbf{x}_{n}\right\}$ and a distance metric $d(\cdot, \cdot)$. For example, this can be a finite subset of $m$-dimensional cube, $X \subset\langle-\gamma, \gamma\rangle^{m} \subset \mathbb{R}^{m}$, and the Euclidean distance. Let $\mathrm{NGH}\left(\mathbf{x}_{i}\right)$ be a list of neighbors of the point $\mathbf{x}_{i}$, sorted in an ascending order according to $d(\cdot, \cdot)$. Further, let $G_{k}=\{X, E\}$ be the k-Nearest Neighbor (k-NN) graph of $X$, i.e.:

$$
\left(\mathbf{x}_{i}, \mathbf{x}_{j}\right) \in E \Leftrightarrow \mathbf{x}_{j} \in\left\{\mathrm{NGH}_{l}\left(\mathbf{x}_{i}\right): l=1 \ldots k\right\}
$$

where $\mathrm{NGH}_{l}\left(\mathbf{x}_{i}\right)$ is the $l$-th element of the list $\mathrm{NGH}\left(\mathbf{x}_{i}\right)$.

The Mutual Nearest Neighbor distance measure, originally proposed in [7, estimates proximity between a pair of points on the basis of their rankings in mutual k-NN lists. In particular, for a pair of points $\mathbf{x}_{i}, \mathbf{x}_{j} \in X$, such that:

$$
\mathbf{x}_{i}=\mathrm{NGH}_{k}\left(\mathbf{x}_{j}\right) \wedge \mathbf{x}_{j}=\mathrm{NGH}_{l}\left(\mathbf{x}_{i}\right)
$$

the value of the MNN distance measure is equal to:

$$
\operatorname{MNN}\left(X, \mathbf{x}_{i}, \mathbf{x}_{j}\right)=k+l
$$

For clustering purposes, the MNN distance measure has a strong advantage over classical distance metrics, e.g. the Euclidean distance, of being more sensitive to changes in local data density 7]. This is illustrated on the example depicted in Fig. 1. The points form two clusters of different densities (marked by $C_{1}$ and $C_{2}$ ). Suppose, that we would like to identify the clusters by simply comparing the distances between the points. A straightforward approach would be to identify the connected components within the data set, assuming that any two points are connected when the distance between them is smaller than some threshold value $\varepsilon$. Using the Euclidean distance, it is impossible to choose a proper threshold value $\varepsilon$. For $\varepsilon \leq 2$ each point from the cluster $C_{1}$ will be assigned to a separate, artificial cluster. On the other hand, for every 
$\varepsilon>2$ the whole data set will be assigned to a single cluster. Now, consider the MNN distance measure. For every two points from the cluster $C_{1}$ that are adjacent to each other and are placed in the same column (e.g., points $a$ and $b$ in Fig. 1) or the same row (e.g., points $b$ and $c$ in Fig. 1) the value of the MNN distance measure is equal to 2 . The same situation occurs in the cluster $C_{2}$. However, the value of MNN distance measure between the points $x$ and $y$ is equal to: $\operatorname{MNN}\left(C_{1} \cup C_{2}, x, y\right)=4$. We can clearly see this from the lists of the nearest neighbors of those two points: $\operatorname{NGH}(x)=\left[\left\{x_{n 1}, x_{n 2}, y\right\}, y_{n 2}, \ldots\right]$ and $\operatorname{NGH}(y)=\left[\left\{y_{n 1}, y_{n 2}\right\}, y_{n 3},\left\{x, y_{n 4}, y_{n 5}\right\}, \ldots\right]$. Consequently, this data set can be properly clustered with the threshold value for the MNN distance measure $\varepsilon=3$.

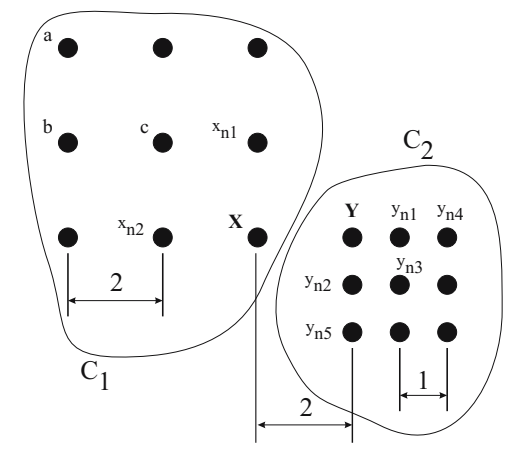

Fig. 1. An example data set, made of two clusters that cannot be discovered using only the Euclidean metric but can be found with the MNN distance measure

\subsection{Estimating Proximity in Multidimensional Spaces with Sparse Shared Nearest Neighbor Graphs}

Euclidean metric is not suited for estimating proximity of points in highdimensional spaces (see e.g. [8]). A proximity measure that is better suited for multidimensional data was proposed in [6]. In this paper, proximity between a pair of points was defined to be the number of neighbors they share. We employ this idea in a slightly modified manner. Consider a k-NN graph $G_{k}=(X, E)$ of the input data set $X$. A graph $S_{k}=(X, E, W)$ in which weights given by:

$$
w_{i j}=\#\left\{\mathbf{x}_{s} \in X \backslash\left\{\mathbf{x}_{i}, \mathbf{x}_{j}\right\}:\left(\mathbf{x}_{i}, \mathbf{x}_{s}\right) \in E \wedge\left(\mathbf{x}_{j}, \mathbf{x}_{s}\right) \in E\right\}
$$

are assigned to edges $\left(\mathbf{x}_{i}, \mathbf{x}_{j}\right) \in E$, is called the Shared Nearest Neighbor graph of $X$. Provided that the number of shared neighbors depends on how close the points are (which is a reasonable assumption), their proximity can be defined in the following way:

$$
d_{S_{k}}\left(\mathbf{x}_{i}, \mathbf{x}_{j}\right)=k-w_{i j}
$$

The measure $d_{S_{k}}\left(\mathbf{x}_{i}, \mathbf{x}_{j}\right)$ is well defined only if both the edges $\left(\mathbf{x}_{i}, \mathbf{x}_{j}\right)$ and $\left(\mathbf{x}_{j}, \mathbf{x}_{i}\right)$ belong to $S_{k}$. If this is not the case, we consider the $d_{S_{k}}\left(\mathbf{x}_{i}, \mathbf{x}_{j}\right)$ to be infinite. 
The SNN graph can be used to establish a strong neighborhood relationship in $X$ [2]. This is done by removing from $S_{k}$ all edges $\left(\mathbf{x}_{i}, \mathbf{x}_{j}\right) \in E$ for which $w_{i j}<t$, where $t$ is a threshold value. In the resultant graph, denoted by $S_{k}^{t}$, edges connect strong neighbors. Relation defined in this way has an advantage of being relatively immune to background noise. In particular, if a sufficiently high threshold value $t$ is chosen, the noise points that lie outside of high density regions (i.e. clusters) will not have any strong neighbors.

\section{Clustering Method Based on the SNN Graph and the MNN Distance Measure}

The pseudo-code of CWNN is presented as Algorithm 1, First, the SNN graph $S_{k}$ of the input data set is build and sparsified using a threshold value $t$. The resultant sparse SNN graph $S_{k}^{t}$ is used to construct the set of core points $X_{c}$, i.e. set of data points that have more than $t_{d}$ strong neighbors within a sphere of radius $\varepsilon_{n}$. It is assumed, that the core points span regions of data space with relatively dense distribution of data points. In the next step, a graph $G_{c}$ of core points is created, in which points $\mathbf{u}, \mathbf{v} \in X_{c}$ are connected if and only if the SNN distance measure $d_{S_{k}}(\mathbf{u}, \mathbf{v})<\varepsilon$ and the MNN distance measure $\operatorname{MNN}_{S_{k}}\left(X_{c}, \mathbf{u}, \mathbf{v}\right)<t_{m}$. Here, $\mathrm{MNN}_{S_{k}}$ denotes the MNN distance measure calculated over the proximity measure derived from the SNN graph $S_{k}$ (eq. 5). To locate origin of clusters, CWNN identifies connected components in $S_{k}$. Finally, CWNN evaluates each of the non-core data points $\mathbf{x}_{i}$ and if $d\left(\mathbf{x}_{i}, \mathbf{c}\right)<\varepsilon_{n}$, where $\mathbf{c} \in X_{c}$ is the core point that is closest to $\mathbf{x}_{i}$, then the point $\mathbf{x}_{i}$ is assigned to the cluster represented by $\mathbf{c}$. Otherwise, $\mathbf{x}_{i}$ is assigned to the noise cluster $C_{N}$.

Our approach employs the MNN distance measure for constructing connected components in the graph of core points. To strengthen this measure against background noise it is calculated over proximity measure derived from the SNN graph. As the MNN distance measure is effective in identifying local changes in data density (see Section 1.1), gradients in the data density should split the graph of core points into a number of connected components, each one with a more uniform density distribution. Consequently, the clustering structure should reveal more information about the analyzed data set. Number of clusters constructed by CWNN is equal to the number of connected components in the graph of core points. This is controlled by two threshold values: $t_{m}$ for the MNN distance measure and $\varepsilon$ for the SNN distance measure. CWNN do not assume any particular geometry of the clusters. In principle, shapes of clusters depend only on the shapes of connected components.

Number of points assigned to the noise cluster depends on two factors: the number of core points identified by the algorithm and the threshold value $\varepsilon_{n}$. The threshold $\varepsilon_{n}$ specifies the maximum distance $d(\cdot, \cdot)$ between a given point $\mathbf{x}_{i}$ and its nearest core point $\mathbf{c}$, which still allows for assigning $\mathbf{x}_{i}$ to the cluster represented by $\mathbf{c}$. The number of core points depends mainly on the distribution of density within the data set. However, setting a broader initial neighborhood 


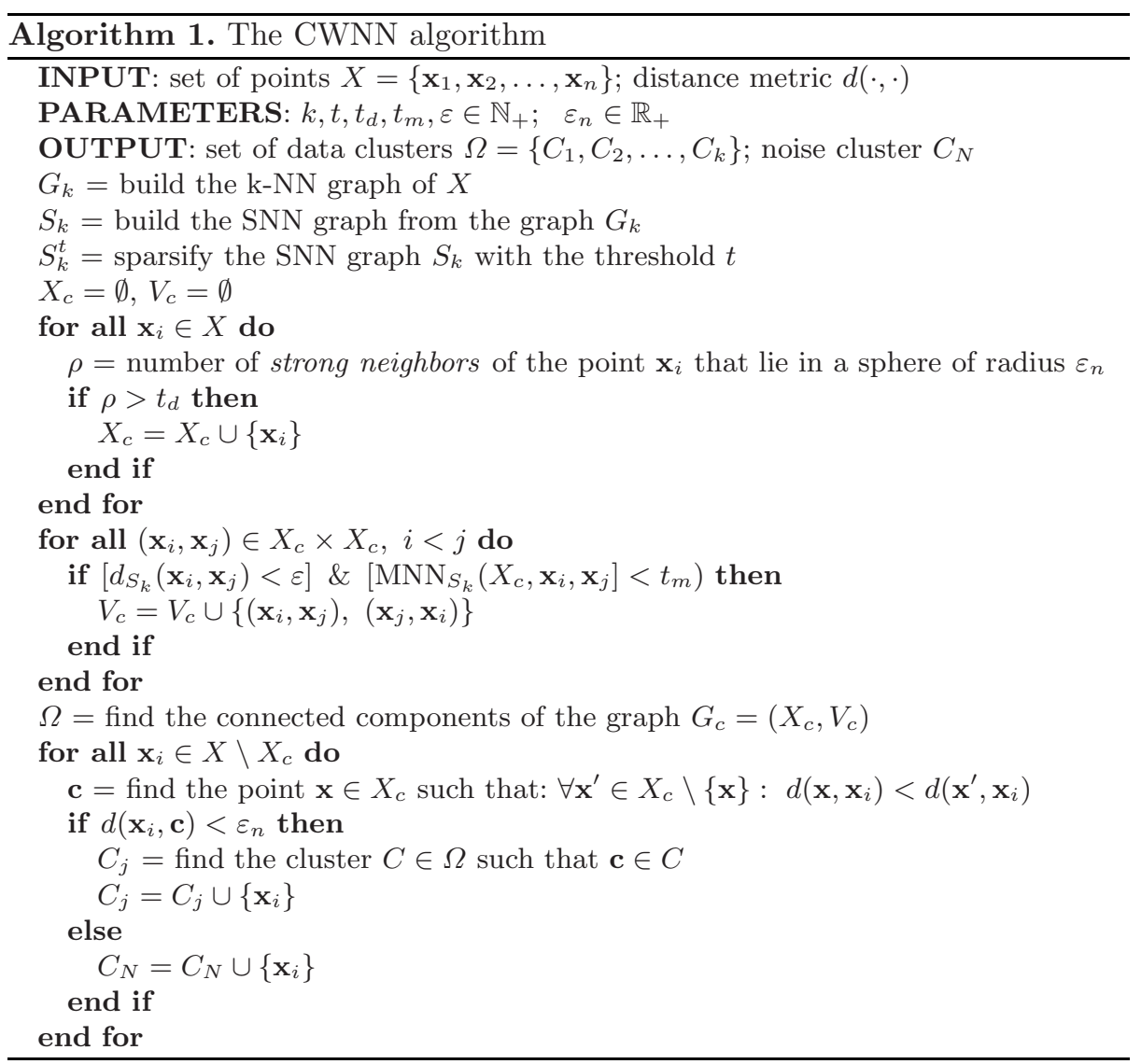

(i.e., higher number of neighbors in the k-NN graph) or decreasing the number of required strong neighbors, $t_{d}$, will increase the number of core points.

The computational complexity of the first part of CWNN, i.e. identification of core points, is $O\left(n^{2} \log n+n k \log k\right)$ due to construction of the k-NN graph and counting of shared neighbors. The computational complexity of the remaining part of the algorithm is $O\left(m^{2} \log m+m \cdot n\right)$, where $m$ is the number of core points. However, the number of core points is smaller than the total number of points: $m \leq n$. Consequently, the computational complexity of the whole CWNN algorithm is approximately $O\left(n^{2} \log n\right)$. The memory complexity is $O\left(n^{2}\right)$.

\section{$3 \quad$ Experimental Results}

Three data sets were used to demonstrate the effectiveness of CWNN. The first one, further called Chameleon data set, was taken from 4. The second one is a synthetic three-dimensional test set, further called tube data set. Third test set, i.e. microcalcification data set, consists of feature vectors constructed by the 
Table 1. Parameters of CWNN used for clustering of the test data sets

\begin{tabular}{ccccccc}
\hline & \multicolumn{5}{c}{ Parameter } \\
\cline { 2 - 7 } & $k$ & $t$ & $t_{d}$ & $t_{m}$ & $\varepsilon$ & $\varepsilon_{n}$ \\
\hline Chameleon data set & 100 & 75 & 4 & 20 & 25 & 10.0 \\
Tube data set & 250 & 180 & 13 & 15 & 115 & 59.0 \\
Microcalcification data set & 1600 & 1100 & 275 & 1900 & 1050 & 2.19 \\
\hline
\end{tabular}

authors during work on an algorithm for detecting suspicious lesions in digital mammograms 9]. Parameters used for clustering the test sets are given in Table 1 . To set the value of these parameters we used the following heuristic. First, we set the value for the number of neighbors in the $\mathrm{k}-\mathrm{NN}$ graph. This graph should reveal local properties of the data set. Therefore, we use a small fraction, i.e. between $1 \%$ to $2 \%$, of the total number of points for this parameter. The upper value is used for multidimensional data. Next, we construct the histogram of the number of shared neighbors in the k-NN graph and locate its maximum. The first minimum following the maximum corresponds to the number of shared nearest neighbors above the most frequent one. We set the parameter $t$ to a value near this minimum, ensuring that strong neighborhood relationship connect only the truly close points. In the next step, we construct the histogram for the number of strong neighbors. This histogram will usually have a peak corresponding to the background noise followed by peaks corresponding to clusters. We set the parameter $t_{d}$ to a value after the peak from noisy background, ensuring correct noise identification. The value for $\varepsilon_{n}$ is set by evaluating all non-noise points. For each such point we calculate the distance to its furthest strong neighbor. The histogram of these distances is used to identify most frequent values and $\varepsilon_{n}$ is set close to them. The parameters $\varepsilon$ and $t_{m}$ are set by inspecting minimal spanning trees of core points constructed using $d_{S_{k}}(\cdot, \cdot)$ and $\operatorname{MNN}_{S_{k}}\left(X_{c}, \cdot, \cdot\right)$ respectively. Again, we construct histograms of edge lengths in these trees. The minima in these histograms correspond to edges connecting clusters. First such minimum is usually a good choice for the value of underlying parameter. Subsequent minima can be used if a more coarse grained clustering is desirable.

Comparative tests. In [4] four two-dimensional data sets were used for evaluation of the Chameleon algorithm, three of which contain background noise. We applied CWNN to these three noisy data sets and in each case were able to obtain the correct clustering. For the lack of space we will report only the result for the hardest case (in [4] it is called $D S_{4}$ ).

The Chameleon data set is pictured in Fig. 2a. The clustering given by CWNN is presented in Fig. 2b. As we can see, CWNN was able to remove the background noise while preserving the bona fide clusters. We should note here, that these clusters differ in densities. Furthermore, the density of the rectangular cluster is near the density of the noise. In addition, clusters lay close to each other. In particular, triangle-like clusters are nearly adjacent. Nevertheless CWNN did succeed in clustering this data set. In comparison, according to [4, DBSCAN, a well known density clustering method, is unable to identify the correct clustering 
(a)

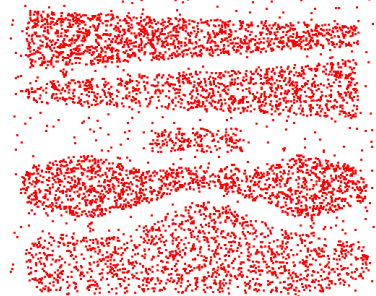

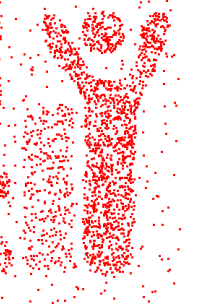

(b)

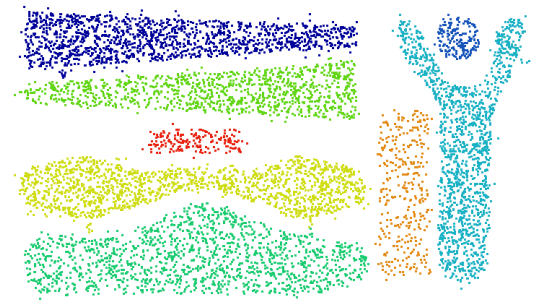

Fig. 2. (a) Two-dimensional Chameleon data set. (b) Clustering of the Chameleon data set obtained with CWNN algorithm.

in this data set. Another density clustering method, CURE, is also reported to fail on this data. Additional results provided on a web page referenced in 4 show that CLARANS 10, ROCK, and group average hierarchical clustering are all unable to correctly cluster the Chameleon data set. The Chameleon algorithm itself managed to identify the genuine clusters in this data. However, this algorithm has no explicit noise removal technique. Therefore, in addition to the bona fide clusters Chameleon constructed additional, spurious clusters out of the background noise, which is evident on the Fig. 6 in [4].

Tube data set. The tube data set is pictured in Fig. 37. It consists of two cubical clusters, namely cluster A and cluster B, surrounded by a variable density background noise. Density of data points in the clusters is five times greater than the density of the surrounding noise. The density of the noise itself increases linearly from the right to the left end of the tube, resulting in 4-fold difference between the ends.

Result of clustering the tube data set is pictured in Fig. 3 $\mathrm{b}$. The two biggest clusters found by CWNN are depicted in Fig. 3. CWNN managed to identify $78.8 \%$ of noise points (i.e. 16,207 out of 20,560). From the 577 data points in the cluster A, 516 were found (89.4\%). In cluster B, out of the 1078 data points, 1075 points were found $(99.7 \%)$. CWNN was therefore successful in discovering both clusters. Some artificial clusters were created from the background noise, near cluster B. This is a consequence of the noise density near left end of the tube being comparable with the density of the cluster A. Therefore, assignment of the whole noisy background to the noise cluster would result in lost of the cluster A. Yet, cluster B was not merged with any of the artificial clusters (see Fig. 35). Gradient of the data density at the border of cluster B, that is well preserved in the set of core points, increases the value of the MNN distance measure between core points in the cluster and core points in the background. This prohibits merging of artificial clusters with cluster B.

Microcalcification feature vectors data set. The microcalcification data set contains feature vectors describing suspicious regions of interest (ROIs) found in 200 high-resolution digital mammograms from the DDSM database [1]. Analysis of such data sets is an important step in design and implementation of 


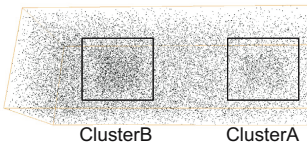

(a)

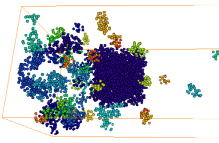

(b)

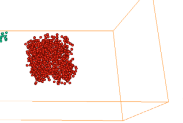

(c)

Fig. 3. (a) Three-dimensional tube data set containing two clusters enclosed by noisy background. (b) Clustering of the tube data set obtained with CWNN algorithm. (c) Two biggest clusters found by CWNN.

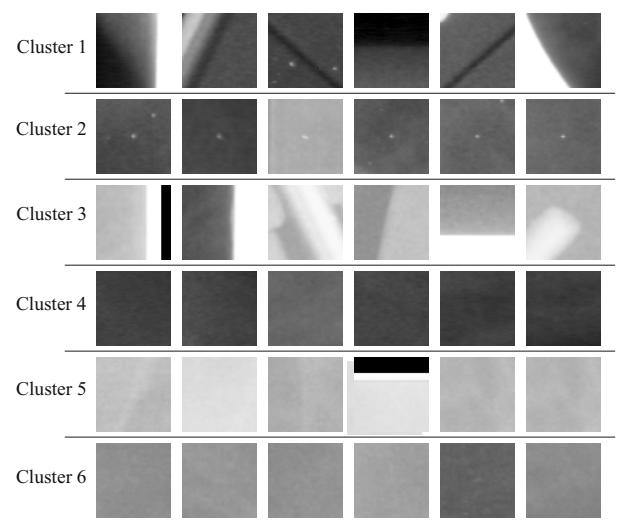

Fig. 4. Example mammogram regions of interest (ROIs), corresponding to feature vectors that belong to different clusters discovered by CWNN

computer aided detection (CAD) systems. Moreover, CAD systems for screening mammography are among most heavily researched computerized detection techniques, owing to difficulties in recognizing early cancer symptoms on mammogram images. Thus, microcalcification data set is an example of rather important class of biomedical data.

Each mammogram ROI is described by 27 pixel intensity features, such as: entropy, contrast, moments of a brightness' histogram, and other. As the algorithm used for initial selection of ROIs was tuned for high sensitivity, a large number of false-positive detections was made. Feature vectors of false-positive ROIs constitute the noise in the data set. Additional details are given in [9].

Out of approximately 93,300 data points in the microcalcification data set, approximately 53,000 (i.e. 57\%) were assigned to noise by CWNN. Six clusters were constructed from the remaining data points. Example ROIs corresponding to feature vectors selected randomly from the six discovered data clusters are presented in Fig. 4. As it can be seen, clusters number 4, 5 and 6 contain flat ROIs characterized by low image contrast. The ROIs differ in the average brightness. Cluster no. 1 contains ROIs with linear structures, usually on a dark background. The regions of interest from the cluster no. 3 contain similar structures but on a brighter background. Finally, cluster no. 2 contains round, punctate occlusions resembling small microcalcifications. 


\section{Implementation Notes}

We have implemented parallel versions of the most costly routines in CWNN, namely construction of the k-NN and sparse SNN graphs and calculation of the MNN distance measure. Parallel version of these routines were designed for shared memory machines and implemented using the OpenMP1 1 standard.

Parallelization of the k-NN graph construction is straightforward. In particular, the first outer loop of this routine runs over all data points, for each one calculating the distances to the points with greater indices. There is no data dependencies between the iterations of this loop and thus they can be directly split between the threads. In the next step, for each point the distances to the remaining points are sorted in an ascending order. Loop performing this sorting can also be parallelized by direct splitting between the threads.

The routine for constructing the sparse SNN graph contains two nested loops. The outer loop runs over all data points. In the i-th iteration of the outer loop, the inner loop runs over nearest neighbors $\mathbf{y} \in \mathrm{NGH}\left(\mathbf{x}_{i}\right)$ of the point $\mathbf{x}_{i}$, assessing for each of them whether $x_{i} \in \mathrm{NGH}(\mathbf{y})$ and counting shared neighbors. These operations require read-only access to the k-NN graph and therefore do not impose data dependencies. However, in rare cases threads can compete for write access to the weights matrix of the SNN graph. We efficiently solved this issue by employing a small hash table of locks that protects elements of the weights matrix. This enables splitting of the outer loop between the threads.

(a)

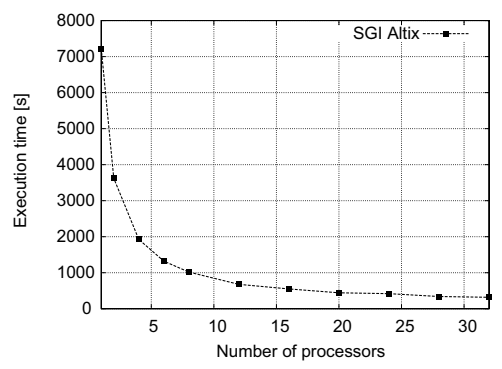

(b)

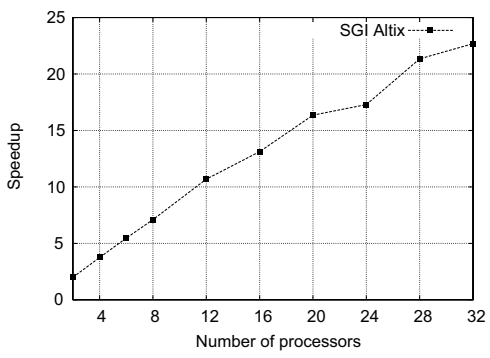
Fig. 5. The execution time (a) and speedup (b) of the parallel implementation of
CWNN algorithm. The results were obtained on the microcalcification data set.

Calculation of the MNN distance measure requires read-only access to the weighted graph of core points. The edge weights are the distance measures derived from the SNN graph (see Sections 1.2 and2). Write access is needed only for the array storing the results. Therefore, we can employ a parallelization strategy similar to the one used in distance calculation during construction of the k-NN graph.

To illustrate the efficiency of the parallelization scheme, benchmark runs on the microcalcification data set were made. The tests were carried out on the SGI

\footnotetext{
1 www. openmp.org
} 
Altix platform, equipped with $1.5 \mathrm{GHz}$ Intel Itanium 2 processors and running Linux operating system. The results are presented in Fig. 5. As we can see, the algorithm scales almost linearly for the number of processors between 2 and 32 .

\section{Future Work}

In the current setup, CWNN can be applied to various types of data provided that a distance metric $d(\cdot, \cdot)$ is available for them. However, the performance of the algorithm in such cases needs further evaluation, which will be the focus of our future research. Another issue to be studied thoroughly are the methods for estimating the initial values for the CWNN parameters. Although our experience shows that with a help of simple heuristic reasonable values for these parameters can be established in few trial runs, an automatic method would make the algorithm more user-friendly.

Acknowledgements. The authors are grateful to the Professor Witold Dzwinel for his valuable comments. This work was partly founded by the Polish Committee for Scientific Research (KBN) grants no. 3T11F01030 and 3T11F01930.

\section{References}

1. Ester, M., Kriegel, H., Sander, J., Xu, X.: A density-based algorithm for discovering clusters in large spatial databases with noise. In: Proceedings of the 2nd International Conference on Knowledge Discovery and Data Mining, pp. 226-231. AAAI Press, USA (1996)

2. Ertöz, L., Steinbach, M., Kumar, V.: Finding clusters of different sizes, shapes, and densities in noisy, high dimensional data. In: Proceedings of the Third SIAM International Conference on Data Mining, San Francisco, CA, USA, vol. 47 (2003)

3. Guha, S., Rastogi, R., Shim, K.: Cure: an efficient clustering algorithm for large databases. In: Proceedings of the 1998 ACM SIGMOD international conference on Management of data, pp. 73-84. ACM Press, New York (1998)

4. Karypis, G., Han, E., Kumar, V.: Chameleon: hierarchical clustering using dynamic modeling. IEEE Computer 32(8), 68-75 (1999)

5. Guha, S., Rastogi, R., Shim, K.: Rock: A robust clustering algorithm for categorical attributes. Information Systems 25(5), 345-366 (2000)

6. Jarvis, R., Patrick, E.: Clustering using a similarity measure based on shared near neighbors. IEEE Transactions on Computers 22(11), 1025-1034 (1973)

7. Gowda, K., Krishna, G.: Agglomerative clustering using the concept of mutual nearest neighborhood. Pattern Recognition 10, 105-112 (1978)

8. Aggarwal, C., Hinneburg, A., Keim, D.: On the surprising behaviour of distance metrics in high dimensional space. In: Van den Bussche, J., Vianu, V. (eds.) ICDT 2001. LNCS, vol. 1973, pp. 420-434. Springer, Heidelberg (2000)

9. Boryczko, K., Kurdziel, M.: Recognition of subtle microcalcifications in highresolution mammograms. In: Proceedings of 4th International Conference on Computer Recognition Systems, Advances in Soft Computing, pp. 485-492 (2005) 
10. Ng, R., Han, J.: Clarans: A method for clustering objects for spatial data mining. IEEE Transactions on Knowledge and Data Engineering 14(5), 1003-1016 (2002)

11. Heath, M., Bowyer, K., Kopans, D., Kegelmeyer, W., Moore, R., Chang, K., Munishkumaran, S.: Current status of the digital database for screening mammography. In: Digital Mammography, pp. 457-460. Kluwer Academic Publishers, Dordrecht (1998) 